\title{
Gauss Map and Local Approach of Isoparametric Surfaces in Lorentz and Euclidean Space
}

\author{
Emre Öztürk ${ }^{1}$ \\ ${ }^{1}$ Turkish Court of Accounts, Ankara, Turkey
}

\section{Article Info}

Keywords: Gauss map, Isoparametric surface, Linear operator

2010 AMS: $14 J 25,32 A 25$

Received: 5 November 2019

Accepted: 23 April 2020

Available online: 10 June 2020

\begin{abstract}
In this study, we determine the isoparametric surfaces and we give the Gauss map of these surfaces by semi symmetric matrix, in Lorentz space. Also we define any chord property and we show that the surfaces which have the chord property corresponds to isoparametric surfaces. Moreover, we consider the chord property locally and we give some examples in the Euclidean space.
\end{abstract}

\section{Introduction and preliminaries}

Isoparametric surfaces, surfaces with constant principal curvatures, are studied in [1]-[3] in terms of the chord property and helical points of the surface in the Euclidean space. In [4], the unit disk characterized by the following:

Lemma 1.1. The only bounded, smooth and simply-connected plane region whose Szegö kernel coincides with the Cauchy kernel is the disc.

Kerzman and Stein [4] used complex analysis technics related with the chord of the curve and they proved the Lemma above. Then, Boas [5] extended this idea to $n$ - dimensional Euclidean space. Boas gave the following theorem, by the help of Bochner-Martinelli kernel:

Theorem 1.2. Ball is the only bounded $C^{1}$ domain in $\mathbb{R}^{m}$ such that given any two points of the boundary, the chord joining them meets the normals at the two endpoints with equal angles.

Thus, in ([5], Proof of Theorem 2, pp. 277-278), the chord property idea of [4] extended to the hyperspheres. Moreover, Boas [2], extended his study [5], to all isoparametric surfaces in the Euclidean space. He gave such a local characterization theorem for hyperspheres and spherical cylinders and proved that these surfaces satisfy

$$
\langle x-y, \overrightarrow{\nabla f}(x)\rangle=\langle y-x, \overrightarrow{\nabla f}(y)\rangle
$$

where $x, y$ are points on surface and $\overrightarrow{\nabla f}$ is the unit normal (gradient) vector field. Wegner [6], gave the short proof of ([2], Local characterization theorem, p.120). In [1], in the light of [2,5], the equation (1.1) considered on a hypersurface such that a unit normal vector field $G$ is naturally defined on the surface. Such $G$ is called the Gauss map of surface. For any hypersphere, the chord joining any two points on it meets the sphere at the same angle at the two points, that is, the sphere satisfies

$$
\langle y-x, G(x)+G(y)\rangle=0
$$


In [1], the following question considered:

What are the hypersurfaces of Euclidean space that satisfy the (1.2)?

They used algebraic approaches and stated that Gauss map of surfaces which satisfy (1.2) is written as $G(x)=A x+b$ where $A$ is constant symmetric matrix, $b$ is column vector. In [7], some special curves are defined and relations between these curves and isoparametric surfaces are given in Lorentz-Minkowski space. In this study, we are looking for answers of the followings:

What are the hypersurfaces of Lorentz space that satisfy the (1.2)?

and

What are the hypersurfaces of Euclidean space that satisfy the (1.2) locally?

In Lorentz space, vectors have different causal characters such as if $\langle u, u\rangle>0$ or $u=0,\langle u, u\rangle<0$ and $\langle u, u\rangle=0(u \neq 0)$ then $u$ is called by spacelike, timelike and lightlike (or null) vector respectively. The number of timelike vectors of the orthonormal basis of the vector space is called the index of space and usually denoted by $v$. Through the [8], we give the followings:

Definition 1.3. Let $X=\left(x_{1}, x_{2}, \ldots, x_{n}\right)$ and $Y=\left(y_{1}, y_{2}, \ldots, y_{n}\right)$ be a two vector distinct from zero in $n$ - dimensional real vector space $\mathbb{R}^{n}$. Following inner product,

$$
\langle X, Y\rangle=-x_{1} y_{1}+\sum_{i=2}^{n} x_{i} y_{i}
$$

is called by Lorentzian inner product of $X$ and $Y$, and $\langle$,$\rangle is called metric tensor of vector space. \left(\mathbb{R}^{n},\langle\rangle,\right)$ is called Lorentz space and denoted by $\mathbb{L}^{n}$ or $\mathbb{R}_{1}^{n}$. If $\langle u, v\rangle=0$ implies that $u=0$ for all $v$ where $u, v \in T_{P} \mathbb{R}^{n}$, then $\langle$,$\rangle is called canonical$ non-degenerated inner product with arbitrary index.

Norm of the vector $u \in \mathbb{R}_{1}^{n}$ is given by $\|u\|=\sqrt{|\langle u, u\rangle|}$. Let the index of $n$-dimensional non-degenerated inner product space of $V$ be $1 \leq v \leq n$ and its orthonormal base be $\left\{e_{1}, e_{2}, \ldots, e_{n}\right\}$. Then $\varepsilon_{1}=\varepsilon_{2}=\ldots=\varepsilon_{v}=-1$ and $\varepsilon_{v+1}=\varepsilon_{v+2}=\ldots=\varepsilon_{n}=1$, where $\varepsilon_{i}=\left\langle e_{i}, e_{i}\right\rangle$. Therefore, the diagonal matrix $\left(\delta_{i j} \varepsilon_{j}\right)$ is called by the sign matrix of $V$ such that

$$
\delta_{i j}=\left\{\begin{array}{l}
1, i=j \\
0, i \neq j
\end{array}, 1 \leq i, j \leq n\right.
$$

is Kronecker delta.

Definition 1.4. [8] Let $n \geq 2$ and $0 \leq v \leq n$,

(1) The pseudosphere of radius $r>0$ in $\mathbb{R}_{v}^{n+1}$ is the hyperquadric

$$
\mathbb{S}_{v}^{n}(r)=\left\{P \in \mathbb{R}_{v}^{n+1} \mid\langle P, P\rangle=r^{2}\right\}
$$

with dimension $n$ and index $v$.

(2) The pseudohyperbolic space of radius $r>0$ in $\mathbb{R}_{v+1}^{n+1}$ is the hyperquadric

$$
\mathbb{H}_{v}^{n}(r)=\left\{P \in \mathbb{R}_{v+1}^{n+1} \mid\langle P, P\rangle=-r^{2}\right\}
$$

with dimension $n$ and index $v$.

Definition 1.5. Let $M$ be a hypersurface in the Minkowski space and $\vec{n}$ be a unit normal vector field of $M$. If $\langle\vec{n}, \vec{n}\rangle\langle 0$, $(\langle\vec{n}, \vec{n}\rangle>0)$ then $M$ is said to be spacelike (timelike) surface.

Lemma 1.6. [8] Let $S$ be a shape operator (Weingarten map) and v be a tangent vector on $M . S(v)=-\nabla_{v} \vec{n}$ and for all $P \in M$, linear operator of $S$ is self-adjoint on $T_{P} M$. Here $\nabla$ is Levi-Civita connection on $M$ in $\mathbb{R}_{1}^{n}$ space.

\section{Linear operators and isoparametric surfaces}

An integral operator in complex space $\mathbb{C}^{n}$ is given by

$$
\zeta(f, x)=\int_{a}^{b} f(x) K(t, x) d t
$$


such that $K(t, x)$ is continuous according to parameter $t$ and it is called the kernel of the operator $\zeta$. Suppose that $\mathscr{B}$ is a bounded smooth domain in the complex plane. The Cauchy kernel represents holomorphic functions $f$ in $\mathscr{B}$ in terms of the boundary values on $\gamma$. Here $\gamma$ is the boundary of the domain $\mathscr{B}$. Cauchy integral operator on any $\mathscr{B}$ domain whose bounded by the curve $\gamma$ in complex plane is given by

$$
\zeta(z, w)=\frac{1}{2 \pi i} \int_{z \in \gamma} \frac{f(z)}{z-w} \dot{\gamma}(z) d \sigma(z)
$$

where $w \in \mathbb{C}$ is on $\gamma$. Here $d z=\dot{\gamma}(z) d \sigma, z=\gamma(s)$ unit speed curve and $d \sigma$ is Lebesgue measure (arc lenght). Hence, Cauchy kernel of the $\zeta$ operator is $\frac{1}{2 \pi i} \frac{1}{z-w} \dot{\gamma}(z)$. Similarly the $\mathscr{S}(z, w)$ Szegö integral operator is given by

$$
\zeta(z, w)=\frac{1}{2 \pi i} \int_{z \in \gamma} f(z) \mathscr{S}(z, w) d \sigma(z)
$$

where the kernel is considered the orthogonal projection of $\mathbb{S}: \mathscr{L}^{2}(\gamma, d \sigma) \rightarrow \mathscr{H}^{2}(\gamma)$. Here $\mathscr{H}^{2}(\gamma)$ is closed subspace of $\mathscr{L}^{2}(\gamma, d \sigma)$ of boundary values of holomorphic functions in $\mathscr{B}$. It is easy to see that $\mathbb{S} \zeta=\zeta$ holds identically and the curve $\gamma$ satisfy

$$
\langle\gamma(t)-\gamma(s), T(t)-T(s)\rangle=0
$$

where $T$ is the unit tangent normal vector field of the curve. It follows from the definitions above and (2.1) that $\gamma$ is non-null hyperbolic curve in Lorentz plane.

Now we extend the chord idea to the surfaces in the high dimensions and we give some characterizations about these surfaces by the help of Gauss map itself, in terms of [1] and [7], in Lorentz space. Throughout this chapter, the metric tensor will be considered as a Lorentzian unless otherwise mentioned. Let us give the following definition first.

Definition 2.1. Let $M$ be a non-null hypersurface and $G$ is Gauss map of $M$. If

$$
\langle Q-P, G(P)+G(Q)\rangle=0
$$

for all $P, Q \in M$ then, $M$ is called by $\mathbf{G}$-hypersurface.

Theorem 2.2. Let $M$ be a $\mathbf{G}$-hypersurface. Gauss map of this surface is given by

$$
G(x)=A x+b
$$

where $A$ is the semi-symmetric matrix and $b \in E_{1}^{n}$ column vector.

Proof. Let the hypersurface $M$ fully lies in space and consider the points $y_{0}, y_{1}, \ldots, y_{n}$ on $M$ such that $\left\{y_{j-} y_{0} \mid 1 \leq j \leq n\right\}$ spans $\mathbb{R}_{1}^{n}$. Similar to [1], we find

$$
A^{T}=\varepsilon\left(B_{j} A_{j}^{-1}\right) \varepsilon
$$

where $\varepsilon=\operatorname{diag}(-1,1, \ldots, 1)$ is the sign matrix, $A_{j}$ and $B_{j}$ are the $n \times n$ matrices that accepts the $y_{j}-y_{0}$ and $G\left(y_{j}\right)-G\left(y_{0}\right)$ as $j$-column respectively. Also $b=\sum_{k=1}^{n} b_{k} \alpha_{k}$ such that $b_{k}$ is given by

$$
\left(\begin{array}{cccc}
\left\langle\alpha_{1}, \alpha_{1}\right\rangle & \left\langle\alpha_{1}, \alpha_{2}\right\rangle & \ldots & \left\langle\alpha_{1}, \alpha_{n}\right\rangle \\
\left\langle\alpha_{2}, \alpha_{1}\right\rangle & \ddots & & \left\langle\alpha_{2}, \alpha_{n}\right\rangle \\
\vdots & & \ddots & \vdots \\
\left\langle\alpha_{n}, \alpha_{1}\right\rangle & \left\langle\alpha_{n}, \alpha_{2}\right\rangle & \ldots & \left\langle\alpha_{n}, \alpha_{n}\right\rangle
\end{array}\right)\left(\begin{array}{c}
b_{1} \\
b_{2} \\
\vdots \\
b_{n}
\end{array}\right)=\left(\begin{array}{c}
c_{1} \\
c_{2} \\
\vdots \\
c_{n}
\end{array}\right)
$$

where $\alpha_{j}=y_{j}-y_{0}$ and $c_{j}=\left\langle G\left(y_{0}\right), y_{0}\right\rangle-\left\langle G\left(y_{j}\right), y_{j}\right\rangle$. Hence we write

$$
c_{j}=\left\langle\alpha_{j}, \alpha_{1}\right\rangle b_{1}+\left\langle\alpha_{j}, \alpha_{2}\right\rangle b_{2}+\cdots+\left\langle\alpha_{j}, \alpha_{n}\right\rangle b_{n}
$$

It follows from (2.2) and (2.3) that

$$
G(x)=A x+b .
$$


We note that $A$ is constant matrix (see [1]). Now we prove that $A$ is semi-symmetric matrix. Let $X, Y \in T_{x} M$ be a tangent vector. Due to Lemma 1.6

$$
\nabla_{X} G(X)=A X=-S(X)
$$

and

$$
\langle S(X), Y\rangle=\langle X, S(Y)\rangle
$$

It follows from (2.4) and (2.5) that

$$
\begin{aligned}
\langle-A X, Y\rangle=\langle X,-A Y\rangle & \Leftrightarrow\langle A X, Y\rangle=\langle X, A Y\rangle \\
& \Leftrightarrow\left\langle X,\left(\varepsilon A^{T} \varepsilon\right) Y\right\rangle=\langle X, A Y\rangle \\
& \Leftrightarrow A=\varepsilon A^{T} \varepsilon
\end{aligned}
$$

which is intended.

Theorem 2.3. Let us assume that hypersurface $M$ have diagonalized shape operator. For non-null hypersurface M, the following statements are equivalent:

i) $M$ is the $\boldsymbol{G}$-hypersurface.

ii) $M$ is an isopametric surface.

iii) $M$ is the open part of non-null hyperplane, pseudosphere, pseudohyperbolic space, pseudospherical cylinder or pseudohyperbolic cylinder.

Proof. Let $M$ be a $\mathbf{G}$-hypersurface and $\left\{E_{1}, E_{2}, \ldots, E_{n-1}\right\}$ orthonormal frame on surface such that $E_{i}, 1 \leq i \leq n-1$ are characteristic vectors corresponding to characteristic values $\mu_{i}$ of the shape operator. Hence, $S\left(E_{i}\right)=\mu_{i} E_{i}$ for all $i$. Due to Theorem 2.2, $G(x)=A x+b$ where $A$ is semi-symmetric matrix. It follows from (2.4) that

$$
A E_{j}(x)=-S\left(E_{j}(x)\right)=-\mu_{j}(x) E_{j}(x)
$$

and

$$
\left(A+\mu_{j} I\right) E_{j}(x)=0
$$

In order to the existence of non-zero characteristic vectors

$$
\operatorname{det}\left(A+\mu_{j} I\right)=0
$$

From equation (2.6), it is obvious that $\mu_{i}$ is constant. Therefore, $M$ is an isoparametric surface.

Let $M$ be an isoparametric surface. Let us define

$$
f(x)=\langle A x+b, A x+b\rangle
$$

where $f: \mathbb{R}_{1}^{n} \rightarrow \mathbb{R}$ and $M \subset f^{-1}( \pm 1)$. It follows from (2.7) that

$$
f(x)=\left\langle x, A^{2} x\right\rangle+2\langle x, A b\rangle+\langle b, b\rangle .
$$

By straightforward calculations we get $\overrightarrow{\nabla f}(x)=2 A(A x+b)$. Since the gradient of $f$ and Gauss map $G$ is linear dependent

$$
A(A x+b)=\lambda(x)(A x+b), x \in M
$$

for some real valued $\lambda(x)$ functions. It follows from (2.8) that $(A-\lambda(x) I)(A x+b)=0$ and $\operatorname{det}(A-\lambda(x) I)=0$. Obviously $\lambda(x)$ is constant. Let us consider $V=\{A x+b \mid x \in M\}$ as a characteristic space that corresponding to $\lambda(x)=\lambda$ and $S p\{V\}$ is normal space at $x \in M$. Let us determine the surface $M$ depends on the norm of $V$.

a) Let $\|V\|=1$. In this case $G(x)=b$ is constant and $M$ is the open part of non-null hyperplane.

b) If $\|V\|=n$ then $V=\operatorname{Im}(A)$. For some $r>0$ and $\lambda= \pm \frac{1}{r}$ we have $\left.A\right|_{V}= \pm \frac{1}{r} I$ such that $G(x)= \pm \frac{1}{r} x+b$. Depends on causal character of $S p\{V\}$, we get pseudosphere of $\mathbb{S}_{1}^{n-1}$ or pseudohyperbolic space of $\mathbb{H}_{1}^{n-1}$. 
c) Let $\|V\|=p, 2 \leq p \leq n-1$. Dimension of $V^{\perp}$ orthogonal complement is $n-p$ and $V^{\perp} \subseteq T_{x} M$. In the neighborhood of $x_{0} \in M$, we choose $\left\{E_{1}(x), E_{2}(x), \ldots, E_{n-1}(x)\right\}$ orthonormal frame such that $E_{1}, E_{2}, \ldots, E_{n-p}$ are constant in $V^{\perp}$., we write

$$
V=S p\left\{E_{n-p+1}(x), E_{n-p+2}(x), \ldots, E_{n-1}(x), G(x)\right\}
$$

The tangent subspace spanned by $\left\{E_{n-p+1}(x), E_{n-p+2}(x), \ldots, E_{n-1}(x)\right\}$ is integrable and integral submanifold of $M_{1}$ through the point $x_{0}$ is given as $M_{1}=M \cap\left(x_{0}+V\right)$. Hence $\mathbb{R}_{1}^{n-p}=V^{\perp}$ and $M=M_{1} \times \mathbb{R}_{1}^{n-p}$ where $M_{1}$ is hypersurface in $\mathbb{R}_{1}^{p}$ and Gauss map of $M_{1}$ satisfy the $G_{1}(x)=G(x)$. Besides, $A_{1}=\left.A\right|_{V}$ satisfy

$$
G_{1}(x)=A_{1} x+b
$$

It follows from (2.8) and (2.9) that $A_{1}= \pm \frac{1}{r} I$. So $M_{1}$ is pseudosphere of $\mathbb{S}_{1}^{p-1}(r)$ or pseudohyperbolic space of $\mathbb{H}_{1}^{p-1}(r)$. Hence $M$ is the open part of $\mathbb{S}_{1}^{p-1}(r) \times \mathbb{R}^{n-p}$ or $\mathbb{H}_{1}^{p-1}(r) \times \mathbb{R}^{n-p}$, respectively.

Let us consider isometric immersion $f_{1}: M \rightarrow \mathbb{R}_{1}^{n}$ with respect to $\left[x_{1}, x_{2}, \ldots, x_{n}\right]$ rectangular coordinate system. Let us give

$$
f_{1}\left(x_{1}, x_{2}, \ldots, x_{n}\right)=a_{1} x_{1}+a_{2} x_{2}+\ldots+a_{n} x_{n}=c
$$

where $a_{i}, 1 \leq i \leq n$ are constant coefficients, $x=\left(x_{1}, x_{2}, \ldots, x_{n}\right) \in M$ and $c$ is real number. The Gauss map of this immersion is given by

$$
G(P):=\left.\frac{\overrightarrow{\nabla f}_{1}}{\left\|\overrightarrow{\nabla f}_{1}\right\|}\right|_{P}=\left.\frac{1}{m}\left(-a_{1}, a_{2}, \ldots, a_{n}\right)\right|_{P}
$$

where $P \in M$ and $m=\sqrt{\left|-a_{1}^{2}+a_{2}^{2}+\ldots+a_{n}^{2}\right|}$. It can be easily seen that

$$
\langle Q-P, G(P)+G(Q)\rangle=0
$$

where $P, Q \in f_{1}$. By Definition 1.4, pseudosphere with center $x_{0}$ and radius $r$ is given by

$$
\mathbb{S}_{1}^{n-1}(r)=\left\{x \in \mathbb{R}_{1}^{n}:\left\langle x-x_{0}, x-x_{0}\right\rangle=r^{2}\right\}
$$

Without loss of generality, we can consider $x_{0}=(0,0, \ldots, 0)$ and $x=\left(x_{1}, x_{2}, \ldots, x_{n}\right)$. In this case,

$$
f_{2}\left(x_{1}, x_{2}, \ldots, x_{n}\right)=-x_{1}^{2}+x_{2}^{2}+\ldots+x_{n}^{2}=r^{2}
$$

where $f_{2}: \mathbb{S}_{1}^{n-1} \rightarrow \mathbb{R}_{1}^{n}$ is an isometric immersion. The Gauss map of this immersion is given by

$$
G(P):=\left.\frac{\overrightarrow{\nabla f}_{2}}{\left\|\overrightarrow{\nabla f}_{2}\right\|}\right|_{P}=\left.\frac{1}{r}\left(x_{1}, x_{2}, \ldots, x_{n}\right)\right|_{P} .
$$

By straightforward calculations we get

$$
\langle Q-P, G(P)+G(Q)\rangle=0
$$

where $P, Q \in f_{2}$. Therefore, $\mathbb{S}_{1}^{n-1}(r)$ is $\mathbf{G}$-hypersurface (similarly $\mathbb{H}_{1}^{n-1}(r)$ is $\mathbf{G}$-hypersurface). Moreover, we consider

$$
-x_{1}^{2}+x_{2}^{2}+\ldots+x_{p}^{2}=r^{2}, x_{p+1}=u_{p+1}, x_{p+2}=u_{p+2}, \ldots, x_{n}=u_{n}
$$

where $u_{i}$ are real variables such that $p+1 \leq i \leq n$. By the help of natural Lorentz projection $\pi_{L}: \mathbb{R}_{1}^{p} \times \mathbb{R}_{1}^{n-p} \rightarrow \mathbb{R}_{1}^{p} \hookrightarrow \mathbb{R}_{1}^{n}$ onto $\mathbb{S}_{1}^{p-1}$ we get

$$
G(P)=\left.\frac{1}{r}\left(x_{1}, x_{2}, \ldots, x_{p}, 0, \ldots, 0\right)\right|_{P}
$$

Hence,

$$
\langle Q-P, G(P)+G(Q)\rangle=0
$$

where $P, Q \in \mathbb{S}_{1}^{p-1}(r) \times \mathbb{R}^{n-p}$. Proof is similar for $\mathbb{H}_{1}^{p-1}(r) \times \mathbb{R}^{n-p}$. 


\section{Local isoparametric surfaces in Euclidean space}

Chord property of isoparametric surfaces are examined locally and globally in $[2,5]$. Our point of view to localization is totally different from previous studies. Let us give the following definition and results.

Definition 3.1. Let $M$ be a hypersurface in Euclidean space and $\langle$,$\rangle be the metric tensor of the space. If some points such$ $P, Q \in M$ satisfy

$$
\langle Q-P, G(P)+G(Q)\rangle=0
$$

then, $M$ is called as local isoparametric surface.

Theorem 3.2. The helicoid surface given by

$$
\Phi(s, t)=(0,0, b s)+t(\cos s, \sin s, 0), b \neq 0
$$

is local isoparametric surface if and only if

$$
p_{1}=q_{1} \quad \text { or } \quad p_{2}=-q_{2}
$$

where $\varphi\left(p_{1}, p_{2}\right)=P, \varphi\left(q_{1}, q_{2}\right)=Q, \varphi: U \subseteq \mathbb{R}^{2} \rightarrow \Phi$ and $\left(p_{1}, p_{2}\right),\left(q_{1}, q_{2}\right) \in U$.

Proof. Let us consider the surface $\varphi(U)=\Phi$ and $\varphi: U \rightarrow \Phi$ differentiable map where $U \subseteq \mathbb{R}^{2}$. Let $P, Q \in \Phi$ two points on surface such that $\varphi\left(p_{1}, p_{2}\right)=P$ and $\varphi\left(q_{1}, q_{2}\right)=Q$. By straightforward calculations we get

$$
\Phi_{s}(s, t)=(-t \sin s, t \cos s, b)
$$

and

$$
\Phi_{t}(s, t)=(\cos s, \sin s, 0)
$$

Unit normal vector field $Z$ of helicoid is given by

$$
Z \circ \varphi=\frac{\Phi_{s}(s, t) \times \Phi_{t}(s, t)}{\left\|\Phi_{s}(s, t) \times \Phi_{t}(s, t)\right\|}=\left(-\frac{b}{\sqrt{b^{2}+t^{2}}} \sin s, \frac{b}{\sqrt{b^{2}+t^{2}}} \cos s,-\frac{1}{\sqrt{b^{2}+t^{2}}} t\right) .
$$

Therefore,

$$
G(P)+G(Q)=\left(\alpha_{1}, \alpha_{2}, \alpha_{3}\right)
$$

where

$$
\begin{aligned}
& \alpha_{1}=-\frac{b}{\sqrt{b^{2}+p_{2}^{2}}} \sin p_{1}-\frac{b}{\sqrt{b^{2}+q_{2}^{2}}} \sin q_{1} \\
& \alpha_{2}=\frac{b}{\sqrt{b^{2}+p_{2}^{2}}} \cos p_{1}+\frac{b}{\sqrt{b^{2}+q_{2}^{2}}} \cos q_{1} \\
& \alpha_{3}=-\frac{b}{\sqrt{b^{2}+p_{2}^{2}}} p_{2}-\frac{1}{\sqrt{b^{2}+q_{2}^{2}}} q_{2} .
\end{aligned}
$$

Besides,

$$
Q-P=\left(q_{2} \cos q_{1}-p_{2} \cos p_{1}, q_{2} \sin q_{1}-p_{2} \sin p_{1}, b q_{1}-b p_{1}\right) .
$$

It follows from (3.1) and (3.2) that

$$
\langle Q-P, G(P)+G(Q)\rangle=b\left(\frac{p_{2}}{\sqrt{b^{2}+p_{2}^{2}}}+\frac{q_{2}}{\sqrt{b^{2}+q_{2}^{2}}}\right)\left(\sin \left(q_{1}-p_{1}\right)+\left(p_{1}-q_{1}\right)\right) .
$$

Obviously $\langle Q-P, G(P)+G(Q)\rangle=0$ if and only if $p_{1}=q_{1}$ or $p_{2}=-q_{2}$. By Definition 3.1, $\Phi$ is local isoparametric surface. 
Theorem 3.3. The hyperbolic paraboloid surface given by

$$
\Phi(u, v)=\left(u, v, \frac{v^{2}}{b^{2}}-\frac{u^{2}}{a^{2}}\right), a, b \in \mathbb{R} \backslash\{0\}
$$

is local isoparametric surface if and only if

$$
\frac{a}{b}=\left|\frac{p_{1}-q_{1}}{p_{2}-q_{2}}\right|
$$

where $\varphi\left(p_{1}, p_{2}\right)=P, \varphi\left(q_{1}, q_{2}\right)=Q, \varphi: U \subseteq \mathbb{R}^{2} \rightarrow \Phi$ and $\left(p_{1}, p_{2}\right),\left(q_{1}, q_{2}\right) \in U$.

Proof. By straightforward calculations we get

$$
Z \circ \varphi=\left(-\frac{2 u}{a^{2} \sqrt{1+\frac{4 u^{2}}{a^{4}}+\frac{4 v^{2}}{b^{4}}}}, \frac{2 v}{b^{2} \sqrt{1+\frac{4 u^{2}}{a^{4}}+\frac{4 v^{2}}{b^{4}}}},-\frac{1}{\sqrt{1+\frac{4 u^{2}}{a^{4}}+\frac{4 v^{2}}{b^{4}}}}\right)
$$

and

$$
\langle Q-P, G(P)+G(Q)\rangle=\frac{\left(-b^{2}\left(p_{1}-q_{1}\right)^{2}+a^{2}\left(p_{2}-q_{2}\right)^{2}\right)\left(\sqrt{1+\frac{4 p_{1}^{2}}{a^{4}}+\frac{4 p_{2}^{2}}{b^{4}}}-\sqrt{1+\frac{4 q_{1}^{2}}{a^{4}}+\frac{4 q_{2}^{2}}{b^{4}}}\right)}{a^{2} b^{2} \sqrt{1+\frac{4 p_{1}^{2}}{a^{4}}+\frac{4 p_{2}^{2}}{b^{4}}} \sqrt{1+\frac{4 q_{1}^{2}}{a^{4}}+\frac{4 q_{2}^{2}}{b^{4}}}} .
$$

Hence $\langle Q-P, G(P)+G(Q)\rangle=0$ if and only if

$$
\frac{a}{b}=\left|\frac{p_{1}-q_{1}}{p_{2}-q_{2}}\right|
$$

which is intended.

Let us give the following surface, in the light of [9].

Theorem 3.4. The Viviani ruled surface given by

$$
\Phi(u, v)=\left(\frac{5}{2}+\frac{5}{2} \cos u, \frac{5}{2} \sin v, 5 \sin \frac{u}{2}\right)+4 v\left(1+\cos u, \sin u, 2 \sin \frac{u}{2}\right)
$$

is local isoparametric surface if and only if

$$
p_{1}=q_{1}+4 k \pi, k \in \mathbb{Z}
$$

where $\varphi\left(p_{1}, p_{2}\right)=P, \varphi\left(q_{1}, q_{2}\right)=Q, \varphi: U \subseteq \mathbb{R}^{2} \rightarrow \Phi$ and $\left(p_{1}, p_{2}\right),\left(q_{1}, q_{2}\right) \in U$.

Proof. Similar to previous operations we get

$$
Z \circ \varphi=\left(\frac{\sqrt{2} \sin ^{3} \frac{u}{2}}{\sqrt{3+\cos u}}, \frac{-5 \cos \frac{u}{2}+\cos \frac{3 u}{2}}{2 \sqrt{2} \sqrt{3+\cos u}}, \frac{\sqrt{2} \cos ^{2} \frac{u}{2}}{\sqrt{3+\cos u}}\right)
$$

and

$$
\langle Q-P, G(P)+G(Q)\rangle=\alpha \sin ^{2}\left(\frac{p_{1}-q_{1}}{4}\right)
$$

where $\alpha$ is non-zero constant. Hence $\langle Q-P, G(P)+G(Q)\rangle=0$ if and only if

$$
p_{1}=q_{1}+4 k \pi, k \in \mathbb{Z}
$$

and this completes the proof. 


\section{Conclusion}

In this study, we showed that the Gauss map of isoparametric surfaces is written by $G(x)=A x+b$ where $A$ is semi-symmetric matrix and $b$ is column vector, in Lorentz space. Moreover, in the Euclidean space; we gave the definition of local isoparametric surface, and we examined the some of them such as helicoid, hyperbolic paraboloid and Viviani ruled surface, by different point of view from the previous studies.

\section{References}

[1] D. S. Kim, Y. H. Kim, New characterizations of spheres, cylinders and W-Curves, Linear Algebra Appl., 432 (2010), $3002-3006$.

[2] H. P. Boas, Spheres and cylinders: A local geometric characterization, Illinois J. Math., 28(1) (1984), $120-124$.

[3] Y. H. Kim, K. E. Lee, Surfaces of Euclidean 4-space whose geodesics are W-Curves, Nihonkai Math. J., 4 (1993), $221-232$.

[4] N. Kerzman, E. M. Stein, The Cauchy kernel, the Szegö kernel, and the Riemann mapping function, Math. Ann., 236 (1978), 85-93.

[5] H. P. Boas, A geometric characterization of the ball and the Bochner-Martinelli kernel, Math. Ann., 248 (1980), $275-278$.

[6] B. Wegner, A differential geometric proof of the local geometric characterization of spheres and cylinders by Boas, Math. Balkanica, 2 (1988), 294-295.

[7] E. Öztürk, Y. Yaylı, W-curves in Lorentz-Minkowski space, Math. Sci. Appl. E-Notes, 5(2) (2017), 76-88.

[8] B. O’Neill, Semi-Riemann geometry with applications to relativity, Academic Press. Inc., 1983.

[9] C. Ekici, E. Özüsağlam, M. Çimdiker, E. Öztürk, On the curvatures of Viviani ruled surface, Ciencia e Tecnica Vitivinicola, 29(7) (2014), 449-475. 